\title{
Le Glasgow City Centre Mural Trail : entre affirmation identitaire et « kitschification » culturelle
}

The Glasgow City Centre Mural Trail: Between Identity Affirmation and Cultural

"Kitschification"

\section{Emerence Hild}

\section{(2) OpenEdition}

\section{Journals}

\section{Édition électronique}

URL : http://journals.openedition.org/etudesecossaises/3716

DOI : 10.4000/etudesecossaises. 3716

ISSN : 1969-6337

\section{Éditeur}

UGA Éditions/Université Grenoble Alpes

\section{Édition imprimée}

ISBN : 978-2-37747-275-8

ISSN : $1240-1439$

\section{Référence électronique}

Emerence Hild, « Le Glasgow City Centre Mural Trail : entre affirmation identitaire et « kitschification » culturelle », Études écossaises [En ligne], 21 | 2021, mis en ligne le 31 mars 2021, consulté le 31 mars 2021. URL : http://journals.openedition.org/etudesecossaises/3716 ; DOI : https://doi.org/10.4000/ etudesecossaises.3716

Ce document a été généré automatiquement le 31 mars 2021.

(c) Études écossaises 


\section{Le Glasgow City Centre Mural Trail : entre affirmation identitaire et « kitschification » culturelle}

The Glasgow City Centre Mural Trail: Between Identity Affirmation and Cultural "Kitschification"

Emerence Hild

\section{Introduction}

1 À l'ère du numérique et de la société de consommation, le développement de l'industrie touristique régi par des logiques économiques repose de plus en plus sur des stratégies de marketing identitaire qui s'inspirent de la culture locale pour valoriser un lieu et accroître son attractivité. Si ces stratégies sont particulièrement perceptibles au niveau national, elles s'expriment en réalité à différentes échelles territoriales. Ainsi, pour favoriser la croissance du tourisme urbain, les villes sont amenées à défendre leurs propres politiques culturelles. Ces politiques peuvent alors refléter le choix de soutenir la promotion d'une identité nationale singulière ou au contraire de valoriser une identité locale distincte. En Écosse, la formation progressive et le rayonnement d'une représentation esthétisée de la culture nationale ont permis l'émergence d'une attirance étrangère pour le pays, avec en 2018 près de 15,5 millions de séjours touristiques passés en Écosse. Cette représentation de l'Écosse est inséparable d'une imagerie nationale basée sur des symboles et des clichés largement répandus dans l'imaginaire populaire international. Elle se matérialise dans la multiplication et la commercialisation d'objets et de souvenirs renvoyant à des symboles archétypaux tels que le tartan ou la cornemuse. Mais des résistances locales existent face à la diffusion d'une telle vision de l'Écosse.

2 C'est ce qui semble être le cas à Glasgow, où les politiques culturelles de la municipalité tendent à éviter la reproduction de clichés et de stéréotypes nationaux au profit de la mise en valeur de la culture et des particularités locales. À travers l'étude approfondie 
de l'une des initiatives du city council, cet article vise à mettre en évidence le réseau de tensions qui existe entre les objectifs politiques de la municipalité de Glasgow, ses moyens de mise en œuvre et les impacts culturels et symboliques de cette mise en œuvre. Cette initiative consiste à financer la réalisation de fresques murales dans le centre-ville de Glasgow par des street artists issus de la scène artistique locale. Il ne s'agit pas exactement d'une politique de commande publique dans la mesure où les artistes présentent librement un projet sans répondre à un appel d'offre, mais sont obligés de prendre en compte une liste de critères établie par le City Centre Mural Fund, le comité en charge du projet et de la sélection des œuvres retenues. Le projet intitulé City Centre Mural Trail comprend à ce jour vingt-neuf œuvres réalisées par une dizaine d'artistes au total, en lien dans certains cas avec la galerie d'art Art-Pistol. Le projet, lancé officiellement en 2014 bien que les premières fresques réalisées avec le consentement des autorités locales soient apparues en 2008, est aujourd'hui conçu comme un parcours balisé qui permet de redécouvrir la ville à travers la découverte des fresques. L'objectif annoncé du projet consiste quant à lui à revaloriser le paysage urbain et à améliorer l'expérience des visiteurs de Glasgow. Cette initiative encore récente connaît aujourd'hui un engouement à la fois critique et populaire qui justifie son choix comme objet d'étude.

3 Si pour un regard complètement extérieur les œuvres ne présentent pas de spécificités qui permettent de les distinguer en tant que parties d'un projet global autorisé et financé par les pouvoirs publics, c'est pourtant bien dans ce contexte là que s'inscrit l'analyse qui suit. Elle sera donc fondée sur un dialogue constant entre les œuvres prises individuellement et le projet global dont l'existence est matériellement attestée par la production d'outils promotionnels variés et multi-supports. À travers l'analyse conjointe des fresques et des outils promotionnels mis en œuvre pour les faire connaître, cette étude propose donc d'examiner le projet du City Centre Mural Trail dans son rapport aux pouvoirs publics et à l'identité locale. Il s'agira d'analyser d'une part l'impact de l'intermédiation institutionnelle sur la portée et la signification du projet, et d'autre part la manière dont les œuvres s'intègrent au sein d'un discours et d'une réflexion sur l'identité locale de la ville. Cette étude fera appel au concept de kitsch, conçu à la fois comme catégorie esthétique et comme mode de fonctionnement que l'on cherchera à définir par ses propriétés. Ainsi, dans un premier temps il sera question d'expliciter le choix du street art comme modalité de mise en œuvre des objectifs énoncés par les pouvoirs publics et comme instrument de l'affirmation d'une identité locale forte distincte des représentations populaires de l'Écosse. Dans un second temps, on reconsidèrera la nature du City Centre Mural Trail et on introduira la notion de décor urbain pour démontrer comment, à travers un processus de " kitschification », le projet favorise la création de nouveaux archétypes symboliques.

\section{Régénération urbaine et reconstruction identitaire à Glasgow}

\section{Quand la politique publique repeint la rue}

Pour comprendre la genèse, les enjeux et la signification du City Centre Mural Trail en tant que politique culturelle, il est nécessaire de s'intéresser d'abord au contexte dans lequel il s'insère. En effet, ce projet s'inscrit dans une volonté globale de revalorisation 
de l'environnement urbain mené par le city council. Comme le résume Gilles Leydier, «[la] stratégie municipale de revitalisation du centre-ville s'est construite autour du pari du tourisme urbain et de congrès, et de la promotion de Glasgow comme ville d'art et de culture » (2005). Les écrits qui s'intéressent à la place des politiques culturelles dans la reconstruction de la ville sont nombreux (voir Keating, 1988; Leydier, 2005 ; Paddison, 1993 ; Hall \& Robertson, 2001) et un consensus général établit un lien de causalité entre l'émergence de ces politiques culturelles volontaristes dans les années 1980 et les problèmes économiques et sociaux auxquelles la ville a été confrontée dans les décennies qui ont précédé suite à la désindustrialisation de la région.

5 Au milieu du $\mathrm{xx}^{\mathrm{e}}$ siècle, la ville de Glasgow a connu une période de déclin industriel majeur. Selon Ronan Paddison, "[whether] viewed at the European scale or nationally, Glasgow represents one of the more extreme cases of an industrial city in decline " (1993). Or, cette période de désindustrialisation a refondé la structure et l'identité de la ville à plusieurs niveaux. Ainsi, le déclin industriel et économique de la ville s'est répercuté dans son apparence même avec la fermeture d'entreprises, une dégradation générale de l'habitat, la redistribution spatiale des quartiers défavorisés étendus au-delà du centre-ville (voir Jeannier, 2008) et l'apparition de problèmes sociaux structurels liés au chômage et à la pauvreté. Par conséquent, l'image de la ville en tant qu'imaginaire projeté sur elle depuis l'extérieur s'est également vue modifiée. Les représentations généralement associées à Glasgow se sont progressivement dégradées pour se doter d'une connotation négative, encore perceptible aujourd'hui, si bien que Ronan Paddison affirme :

[It] would be difficult to deny the negative images held of the city by those external to it, either from elsewhere within Scotland and the UK and further afield. These negative stereotypes are linked to its high levels of deprivation, its economic decline, its high crime rates and particularly violent crime, and to secterianism. (2013)

6 Dès les années 1980, les pouvoirs publics ont donc décidé d'engager un processus de régénération urbaine pour attirer une nouvelle population et relancer l'économie locale. Pour répondre au double objectif de réhabiliter la ville - en particulier le centre-ville - et de reconstruire une identité locale positive et attractive, le city council a privilégié un plan stratégique axé autour de politiques culturelles visant à faire de Glasgow un centre événementiel et artistique dynamique. En effet, promouvoir la culture comme mode d'action privilégié devait permettre à la fois de contrer l'image négative de la ville en diffusant celle d'une identité post-industrielle assumée et d'encourager le tourisme pour générer de nouveaux emplois (Paddison, 1993). Ce processus s'est concrétisé dans la mise en œuvre d'un marketing urbain ambitieux, porté par des campagnes publicitaires et des slogans tels que "Glasgow's Miles Better ", et dans la tenue d'événements culturels réguliers et variés, parmi lesquels les événements liés à l'attribution du titre de «Capitale européenne de la culture » en 1990 ou les Jeux du Commonwealth en 2014 (Leydier, 2005).

7 Le projet de revalorisation de la ville de Glasgow amorcé dans les années 1980 s'est inscrit dans le long terme et perdure aujourd'hui. Ainsi les politiques culturelles menées à l'origine pour reconstruire une image de la ville distincte des stéréotypes négatifs qui y étaient traditionnellement associés sont elles-mêmes devenues un élément constitutif de cette nouvelle identité. La ville de Glasgow continue donc de déployer un ensemble d'actions visant à promouvoir son statut de ville de la culture et des arts, parmi lesquelles la commande ou la subvention d'œuvres destinées à 
l'enrichissement de l'environnement urbain. C'est dans cette perspective que nous analysons le City Centre Mural Trail. L'art public, conçu ici comme une performance ou une production artistique réalisée ou située dans l'espace public, peut apparaître comme un mode d'action efficace dans le cadre de la régénération urbaine :

Hall and Robertson $(2001$, p. 7) cite [...] the Policy Studies Institute's summary of the contribution that public art can make to a number of contemporary urban issues: contributing to local distinctiveness; attracting investment; boosting cultural tourism; enhancing land values; creating employment; increasing use of urban spaces; and, reducing vandalism. (Sharp, Pollock \& Paddison, 2005)

Le street art en particulier, offre une modalité d'intervention et d'exploitation de l'espace urbain singulière aux implications complexes. En effet, bien que le street art se soit démocratisé et que " [1]'opinion positive dont bénéficie actuellement le street art [ait] placé les artistes en lieu et place pour jouer le rôle d'auxiliaire dans le processus d'urbanisation par l'animation et la décoration " (Riffaud \& Recours, 2016, $\S 10)$ le choix de ce mode d'expression par les pouvoirs publics relève de stratégies particulières.

8 La visibilité du street art est un élément déterminant dans le cadre des politiques locales. Elle lui permet d'atteindre un large public, non seulement de connaisseurs venus observer les œuvres volontairement mais aussi de passants directement mis en contact avec celles-ci. Dans le cas du City Centre Mural Trail, plusieurs éléments favorisent leur visibilité. Leur monumentalisme tout d'abord: la plupart des œuvres occupent des pans de murs entiers, voire plusieurs bâtiments, comme la fresque Strathclyde University des deux artistes locaux Ejek et Rogue-One qui fait partie des plus grandes fresques murales de Grande-Bretagne. Ce monumentalisme est permis et favorisé par le mode de conception et de production de ces œuvres. La concertation des artistes avec les pouvoirs publics locaux garantit pour les artistes la possibilité de réaliser des fresques de grand format, possibilité non seulement juridique mais aussi matérielle, et pour les pouvoirs publics l'assurance d'œuvres facilement accessibles et visualisables. La promotion de ces œuvres, par le biais d'un site internet et d'une application mobile dédiés ainsi que d'un portfolio imprimé contenant une description des œuvres et un plan pour les situer dans la ville, participe de la même stratégie et contribue également à la visibilité du projet. Enfin, le mode de production de ces fresques, à savoir au grand jour, offre aux riverains la possibilité de voir l'œuvre en construction et de s'approprier les phases successives de son élaboration. Certaines fresques réalisées en direct deviennent leurs propres événements et peuvent être présentées comme tels lors de certaines occasions. Ce fut le cas par exemple pour l'œuvre Wind Power de l'artiste Rogue-One peinte au moment des Journées du patrimoine de 2014. Le Mural Trail devient ainsi progressivement un composant familier et ouvertement valorisé de l'environnement urbain.

9 La deuxième fonction essentielle des œuvres tient dans leur aspect décoratif et ornemental, entièrement assumé puisqu'il s'agit d'un des critères fondamentaux de l'attribution des subventions aux artistes. Les efforts promotionnels réalisés autour du projet présentent de manière systématique les fresques murales comme un moyen de « redynamiser le décor des rues et de redonner vie aux bâtiments et aux terrains désaffectés ». La description de certaines œuvres dans le livret d'information insiste sur la participation du programme à la rénovation physique de la ville. La fresque Fellow Glasgow Residents de Smug, artiste australien basé à Glasgow, a permis de restaurer un mur sur lequel se trouvait "de la peinture écaillée et des graffitis». Hand Shadow 
Puppets de Rogue-One, réalisée dans un passage souterrain, a pour but "d'égayer un lieu qui devenait de plus en plus terne » et The Musician du même artiste "d'ajouter de la couleur et de la créativité aux allées renaissantes de la ville». Ainsi, comme l'indiquent Thomas Riffaud et Robin Recours :

[Le talent des artistes] et leur penchant pour la couleur sont utilisés pour esthétiser la rue et ses interstices. Les œuvres deviennent « un équipement utile » (Fourquet \& Murard, 1973) qui, en plus de décorer, donnent une image positive aux décideurs ayant soutenu le projet. (2016, § 10)

La fonction décorative des œuvres du Mural Trail permet donc de matérialiser l'amélioration infrastructurelle promise par les pouvoirs publics locaux et de justifier le financement de ces réalisations artistiques. C'est pourquoi le choix des emplacements de ces fresques se fait également de manière concertée entre les artistes et le City Centre Mural Fund en charge de l'attribution des financements. Les emplacements, tous dans le périmètre restreint du centre-ville, comprennent ainsi une variété de lieux - rue piétonne, parking, université, etc. - et de supports muraux - façades de magasins ou de bars, pignons d'immeubles, passages souterrains, etc. La diffusion des œuvres à travers l'espace public assure ainsi leur intégration et favorise leur acceptation au sein du paysage urbain.

11 Enfin, le City Centre Mural Trail possède également une dimension ludique, explicitement requise par les pouvoirs publics qui privilégient des œuvres stimulantes qui doivent permettre d'enrichir l'expérience des visiteurs venus à Glasgow. Prises individuellement, certaines fresques répondent à ce critère en instaurant un jeu avec leur environnement et avec le spectateur. On prendra ici pour exemple la fresque Honey I Shrunk the Kids ${ }^{1}$ de Smug. La fresque représente une femme qui occupe toute la surface de la façade latérale d'un immeuble. Penchée sur une loupe, elle semble vouloir attraper quelque chose au sol. Depuis la rue, la fresque laisse penser que la femme observe les passants et cherche à les attraper. Elle invite donc le spectateur à s'amuser de la mise en abyme créée par le jeu de regard établi entre le passant qui observe la femme, qui observe un objet au sol, qui pourrait être le passant lui-même. Pensé comme une nouvelle attraction touristique qui s'intègre dans la liste des "choses à voir » dans la ville ${ }^{2}$, le projet global réunissant toutes les œuvres est conçu comme un sentier de découverte de la ville. C'est pourquoi chacune des œuvres est indiquée sur un plan de la ville disponible en ligne ou à la fin du portfolio imprimé. Celui-ci contient également pour chaque fresque une petite section intitulée "What's nearby" permettant soit de prolonger la découverte de la ville à partir du sentier, soit d'intégrer la découverte des œuvres dans un parcours de visites préalablement défini. Le Mural Trail devient alors un jeu de piste géant et une expérience à part entière.

\section{L'affirmation d'une identité locale forte}

12 Après avoir souligné l'intérêt et les enjeux de l'exploitation du street art par les pouvoirs publics dans le cadre d'un processus plus général de revitalisation urbaine, un élément significatif semble émerger. Si le street art offre un mode d'action privilégié au regard des objectifs de régénération urbaine et de marketing urbain poursuivis par la municipalité, c'est parce que ce mode d'expression et de représentation artistique s'inscrit dans la rue de manière visible et concrète et opère par là même l'ancrage territorial d'une pratique et de productions artistiques. En s'imprégnant sur les murs de la ville, les œuvres s'intègrent à son récit. Dès lors, il est possible de questionner les 
implications sociales du projet, dans la mesure où celui-ci vient mettre en jeu une réflexion autour de l'identité locale de la ville. Comme le soulignent Joanne Sharp, Venda Pollock et Ronan Paddison :

[Public] art is art which has as its goal a desire to engage with its audiences and to create spaces-whether material, virtual or imagined-within which people can identify themselves, perhaps by creating a renewed reflection on community, on the uses of public spaces or on our behaviour within them. (2005)

13 À travers l'étude des choix symboliques et esthétiques des œuvres, il s'agit donc d'analyser la manière dont le projet offre à la fois une plateforme d'expression capable de réinterpréter et de diffuser une certaine vision de la ville et un outil d'identification en tant que médium populaire et urbain.

Pour les pouvoirs publics, le fait de regrouper les fresques au sein d'un livret informatif assure la cohérence du projet et permet d'affirmer l'unité globale des œuvres et de leur visée. Cette proclamation d'une unité collective, préalablement opérée de manière implicite par l'attribution d'un financement dans le cadre d'un programme officiel, justifie l'étude des œuvres comme véhicules d'un message cohérent. Le dialogue des œuvres entre elles et du projet dans son ensemble permet alors de mesurer la portée symbolique du Mural Trail. Il fait apparaître des points de convergence entre les œuvres qui peuvent donc être interprétés. Il est important de rappeler que les artistes sont bien à l'origine des œuvres et proposent eux-mêmes lors de leur candidature au programme de financement un projet intégral bien défini. Si les œuvres sont financées et doivent répondre à un certain nombre de critères, elles ne sont donc pas pour autant le résultat d'un appel d'offre ou d'une commande prescriptive, mais bien le reflet d'une inspiration originale. Toutefois, le fait que le travail des artistes s'inscrive dans un cadre institutionnel signifie qu'ils deviennent de facto les intermédiaires entre un projet politique et le public. Leurs œuvres se lisent donc à la lumière de ce contexte et de leurs modes de production et de diffusion particuliers.

Plus de la moitié des fresques qui constituent le projet font référence de manière plus ou moins explicite à des personnalités ou événements considérés comme représentatifs ou emblématiques de la ville et de son patrimoine. Les personnalités mises en valeur peuvent être anonymes, comme c'est en partie le cas sur la fresque Strathclyde University dont le but est justement de mettre en valeur le travail et les exploits des personnes passées par cette université. Il en va de même pour la fresque The Musician qui, bien qu'elle ne représente qu'un seul individu, se veut d'après l'artiste Rogue-One qui l'a créée le reflet de la scène musicale locale. D'autres fresques au contraire représentent des personnalités célèbres telles que l'acteur Billy Connolly, qui à lui seul est le sujet de trois fresques réalisées à l'occasion de son $75^{\mathrm{e}}$ anniversaire. La fresque The Clutha qui orne les deux côtés du bar du même nom sur plus de 50 mètres reflète la vivacité culturelle du lieu en représentant les visages de personnalités ayant visité cet endroit célèbre. Quant aux fresques Badminton Mural de Guido Van Helten et The Swimmer de Smug, elles ont toutes deux été réalisées dans le cadre des Jeux du Commonwealth de 2014 pour promouvoir cet événement et mettre en avant des sportifs locaux tels que Kieran Merrilees. Cette démarche de glorification de la réussite d'individus célèbres ou anonymes, mais systématiquement identifiés comme membres de la communauté de Glasgow, permet de recréer un imaginaire local au sein duquel les individus sont en mesure de se reconnaître et qui valorise la ville et sa population. 
16 Fellow Glasgow Residents représente sur le mur d'un parking la végétation et les animaux que l'on retrouve en général dans les parcs de la ville : oiseaux, écureuils, etc. Au-delà de son aspect décoratif, cette fresque renverse l'image industrielle de la ville pour célébrer au contraire la nature et la diversité de Glasgow. La fresque intitulée St Mungo et réalisée comme la précédente par l'artiste Smug propose quant à elle un portrait moderne et hyperréaliste du saint patron de la ville. Ce portrait fait référence à un épisode de la vie du saint lors duquel il fit revenir à la vie un rouge-gorge tué par certains de ses camarades pour le faire accuser. Ici, l'homme représenté comme un simple badaud tient sur son doigt l'oiseau, et seule l'auréole discrètement dessinée en arrière-plan renvoie à l'image traditionnelle de la sainteté. Sur d'autres fresques, des symboles plus subtiles ou cachés peuvent aussi être disséminés, comme la canette de Irn Bru, boisson écossaise, dans les mains de Mona Lisa sur la fresque The Gallery qui détourne des œuvres célèbres telles que La Joconde de De Vinci, La Nuit étoilée de Van Gogh ou Les Demoiselles d'Avignon de Picasso.

Chacune des œuvres décrites précédemment se sert donc de symboles pour transmettre une vision particulière de l'identité locale de la ville. Cette identité réinterprétée par l'intermédiaire de la représentation artistique repose sur plusieurs idées : une vision positive de la communauté urbaine, une glorification de l'effort et du dépassement de soi, une célébration de la diversité artistique et culturelle locale, une valorisation de la ville et de la culture urbaine. Or, elle révèle également une volonté politique de différenciation de la ville de Glasgow. En cherchant à affirmer l'identité locale de la ville par le biais de la mise en valeur de ses propres particularismes, le city council fait le choix à travers sa politique culturelle de mettre à distance, voire de rejeter, une identité nationale écossaise dans laquelle le projet de revalorisation de la ville ne peut pas s'inscrire. Il est intéressant à ce titre de noter que dans le plan quinquennal stratégique de développement touristique édité par le City Marketing Bureau en 2011, la volonté de différenciation de la ville de Glasgow par rapport au reste de l'Écosse était clairement exprimée. En effet, la stratégie adoptée dans le document consistait à repositionner la ville «en développant son image positive et singulière ». De fait, le slogan choisit à cette période, "Glasgow: Scotland with Style ", est révélateur de la volonté des pouvoirs publics de s'inscrire dans un discours critique vis-à-vis de l'image kitsch de l'Écosse puisqu'implicitement elle prend ses distances avec une «Scotland without Style». Ainsi, plutôt que de s'intégrer dans le schéma stratégique de développement touristique national basé sur la diffusion d'une imagerie esthétisée de l'Écosse, la ville de Glasgow privilégie un plan local de revalorisation et d'affirmation identitaire.

Le City Centre Mural Trail, officiellement lancé en 2014, s'inscrit clairement dans ce plan stratégique dans la mesure où chacune des fresques mentionnées ci-dessus pioche dans un réseau de symboles évocateurs au niveau local et non national. Il participe également à la diffusion d'une image résolument moderne et urbaine de l'identité locale, distincte des stéréotypes nationaux. Le choix même du street art par les pouvoirs publics indique leur volonté d'inscrire la ville dans une contre-culture postindustrielle. En utilisant un médium qui, par son origine et ses connotations, renvoie à une culture populaire et urbaine, les pouvoirs publics locaux mettent en scène une ville ouverte et inclusive où s'exprime une identité locale forte. 

accessibles et symboliquement significatives. Ces œuvres favorisent ainsi l'émergence d'un esprit de cohésion autour de la revalorisation de la ville de Glasgow, de son histoire et de ses particularismes locaux dans un processus afférent à l'art public :

Key to the creation of social cohesion is the belief that public art, or the processes through which it is produced, is able to create a sense of inclusion. By this token, public art should be able to generate a sense of ownership forging the connection between citizens, city spaces and their meaning as places through which subjectivity is constructed. (Sharp, Pollock \& Paddison, 2005)

Le succès du City Centre Mural Trail en tant que politique culturelle tient peut-être finalement à sa capacité à dépasser ce cadre politique et à s'intégrer dans l'espace commun par un mouvement de patrimonialisation (Riffaud \& Recours, 2016). Grâce à l'engouement critique et populaire qu'il a su susciter, manifeste dans l'obtention de prix $^{3}$, son référencement comme attraction touristique sur plusieurs sites internet, sa mention dans de nombreux blogs de voyageurs, le Mural Trail devient progressivement un nouveau point de repère et d'identification pour la ville de Glasgow. Malgré un avertissement sur la nature provisoire des œuvres de street art en dernière page du portfolio, le fait de les regrouper et de les promouvoir leur confère un statut particulier qui encourage leur protection. À la faveur de cette durabilité acquise dans le paysage urbain, le Mural Trail représente désormais non seulement un atout favorable à un tourisme culturel s'adressant à un public jeune et cosmopolite, mais devient peut-être aussi l'expression et le support d'un sentiment d'appartenance territorial partagé au sein d'une communauté qui se reconnaît dans l'identité projetée par ces œuvres.

\section{« Kitschification » et glissement ontologique du City Centre Mural Trail}

\section{Le décor urbain, équivalent kitsch du street art?}

21 L'analyse menée ci-dessus ouvre des perspectives quant à la capacité des politiques culturelles, et du street art en particulier, à créer du lien social et à favoriser l'industrie touristique. Plusieurs éléments laissent néanmoins transparaître les limites de cette pratique. Après avoir considéré le City Centre Mural Trail sous l'angle de la politique culturelle et des objectifs économiques et sociaux qui sous-tendent le projet, il est possible de l'analyser à partir de son contexte artistique pour souligner les tensions qui émanent de l'institutionnalisation de la pratique artistique à des fins promotionnelles et touristiques. L'analyse qui suit repose sur une conception de la notion de kitsch définie à partir de ses propriétés : esthétique de la surcharge et du trop-plein, disjonction entre fond et forme de l'œuvre, célébration de la beauté du monde et d'une communauté universelle des êtres, familiarité et proximité, transformation de l'œuvre en bien consommable (voir Genin, 2007). L'introduction du concept de «kitschification » utilisé par Christophe Genin dans son ouvrage Le Street Art au tournant et qu'il définit comme "une inversion sémiotique et sémantique : la conversion du dérangeant en mignon, de l'intolérable en décoratif, de l'effaçable en patrimonialisable» (2016) permet de proposer une lecture et une interprétation des propriétés kitsch du City Centre Mural Trail. L'analyse qui suit vise donc à démontrer que 
le projet étudié rentre dans un schéma d'inversions qui met en question sa nature même.

Jusqu'à présent nous avons utilisé le terme de street art pour qualifier les fresques murales qui constituent le Mural Trail. C'est le terme retenu dans les documents édités par le city council, repris dans la presse et généralement accepté par le grand public pour parler de fresques murales peintes dans la rue. Il est toutefois nécessaire de rappeler que ce terme n'est ni neutre ni évident. Il se heurte à des divergences d'interprétation entre des chercheurs qui considèrent que l'étude du street art "nécessite de faire le deuil de notre obsession à mettre les objets dans des boîtes pour tenter de les comprendre» (Riffaud \& Recours, 2016, § 2) et ceux pour qui la difficulté qui existe à définir les pratiques artistiques de l'espace public ne doit pas justifier l'emploi d'un seul terme englobant pour toutes les qualifier indifféremment (Blanché, 2015). Ainsi l'emploi du terme de street art selon les ouvrages va d'une définition universelle de la pratique artistique délocalisée dans l'espace public jusqu'à une définition restrictive qui fait du street art une pratique politique et sociale fondée sur la transgression (des frontières de la propriété, des structures d'autorité, des discours dominants...). Cet article n'a pas vocation à détailler ou à développer ce débat, mais soulève l'existence de ce questionnement car il est révélateur de la complexité inhérente à cette activité polymorphe (Genin, 2015).

S'il semble donc impossible de définir le street art de manière unitaire et d'en délimiter précisément les contours, on peut considérer que le "phénomène social initial » auquel il se réfère :

[...] est l'appropriation sauvage ou vandale de l'espace de circulation extérieur, public ou privé, ou des lieux dévalués (toilettes, impasses, chantiers, taudis, friches industrielles) par des graveurs, scripteurs et dessinateurs qui, de façon anonyme ou chiffrée au départ, déposent dans cet espace des signes urbains [...]. (Genin, 2015, $\S 40)$

Dès lors qu'on considère ce phénomène comme la genèse d'une pratique qui s'est progressivement diversifiée tant dans ses modes de production que dans ses modes de diffusion ou dans son degré de légitimation, il est possible de replacer le City Centre Mural Trail dans une filiation artistique qui permet de considérer les effets de l'intermédiation institutionnelle sur la nature même du projet.

Tout d'abord, il est possible d'affirmer que la légitimation du street art par le bais de son utilisation par les pouvoirs publics le prive de sa dimension contestataire initiale. En d'autres termes, en devenant un outil exploitable par les pouvoirs publics, le street art se dérobe à toutes possibilités de remise en question des discours et des images dominants. Thomas Riffaud et Robin Recours mentionnent le phénomène dans ces termes :

Répondre à un appel à projet pour une fresque consiste très rarement à peindre en toute liberté. La demande publique se satisfait souvent d'œuvres consensuelles qui contestent peu l'espace où elles se trouvent. [...] Comme dans tous les domaines de l'art, l'institutionnalisation a tendance à réduire le potentiel de contestation. (Riffaud \& Recours, 2016, § 11)

Le City Centre Mural Trail illustre à cet égard un glissement du rapport entre pouvoirs publics et street art: les artistes sont financés pour réaliser des fresques à vocation décorative qui viennent valoriser l'image de la ville, en échange de quoi leur travail s'intègre dans un cadre légitime où les œuvres sont reconnues et tacitement protégées. 
Le projet semble en ce sens se rapprocher des catégories établies par Christophe Genin de street art publicitaire et de street art héréditaire (Genin, 2015).

Ce basculement entre le caractère artistique et le caractère publicitaire des œuvres est un basculement générique qui pose la question de l'authenticité des œuvres. Les choix esthétiques deviennent des instruments promotionnels qui ne servent plus seulement des intérêts artistiques mais aussi économiques. Les œuvres sont directement construites autour de la nécessité d'être vues et appréciées. D'où à la fois leur gigantisme et leur réalisme. Comme l'écrit un journaliste, "while these murals are hailed for their photorealism, underground taggers are more likely to use complex 'bubble' or 'widestyle' patterns only decipherable to fellow artists to avoid police detection ${ }^{4}$ ». Les œuvres de street art non autorisées cherchent à créer de la distance en utilisant un mode d'expression complexe, inintelligible aux non-initiés et en s'appropriant des espaces dégradés ou abandonnés. Au contraire, les œuvres du Mural Trail se limitent à un espace balisé, le centre-ville, et fréquenté, puisque touristique. Leur emplacement est directement indiqué sur le plan de la ville fourni par le city council ce qui facilite d'autant plus leur découverte. Ainsi, «alors que l'œuvre gardait ses distances, supportant mal une proximité profanatoire, l'objet kitsch est familier, offert au toutvenant » (Genin, 2007).

En outre, tandis que le street art non autorisé se donne fréquemment pour but de révéler l'existence de problèmes politiques et sociaux, de donner une voix aux minorités exclues, de remettre en question les systèmes établis, le décor urbain magnifie la ville, voire la maquille, et projette l'image d'une prétendue fraternité universelle. Au contre-récit du street art se substitue la fiction du décor urbain : « Kitsch reduces all the complexity, desperation and paradox of human experience to simple sentiment [...].» (Binkley, 2000) Le décor urbain se détourne ainsi des problèmes sociétaux pour promouvoir l'idée d'un bien-être social absolu. Le Mural Trail n'échappe pas à cette dynamique qui vise à se persuader de l'existence d'un "état idyllique " (Genin, 2007) caractéristique du kitsch. Les problèmes auxquels la ville de Glasgow est confrontée - chômage, criminalité, santé publique - et qui en font la ville «la plus malade du Royaume-Uni ${ }^{5}$ » ne transparaissent pas dans les fresques financées par le city council. Parallèlement à l'engouement que crée le Mural Trail, les politiques culturelles de la ville dont il fait partie peuvent ainsi parfois faire l'objet de critiques :

Allied to these debates emerging specifically from Glasgow 1990 as to the identity of the city and whose representation of the new image was being projected, has been an ongoing criticism centring on the image-reality gap-that the projection of the new Glasgow bore little relevance to the realities of social deprivation and poverty concentrated in the city's peripheral estates. (Paddison, 1993)

\section{De l'art au produit culturel}

L'intermédiation institutionnelle dans le cadre du City Centre Mural Trail aboutit à une reconfiguration générique qui pose également la question du contenu sémantique des œuvres. Cette reconfiguration générique pose également la question du contenu sémantique des œuvres. En tant que composantes d'un décor urbain, les œuvres diffusent une représentation biaisée de l'identité locale en occultant certaines réalités et en créant de nouveaux archétypes symboliques. Puisque le décor urbain a une visée touristique, il participe à un mouvement général qui consiste à réduire l'identité locale à une image typique. Ce processus passe notamment par la simplification et la 
marchandisation des attributs considérés comme représentatifs de cette culture spécifique. Dans cette perspective, le décor urbain de la ville de Glasgow n'est pas le simple reflet d'une identité locale mais repose en partie sur l'instrumentalisation de certains symboles qu'il cherche à mettre en valeur.

Les trois fresques dédiées à Billy Connolly s'inscrivent dans ce schéma. Le contexte de production de ces trois fresques diffère des autres œuvres. Àl'occasion du $75^{\mathrm{e}}$ anniversaire de l'acteur, BBC Scotland a commandé la réalisation de trois portraits lui rendant hommage à trois artistes écossais. Chacun renvoie à une image différente de l'acteur: le portrait réalisé par John Byrne représente Billy Connolly de manière réaliste, Rachel MacLean utilise la surcharge symbolique du costume pour célébrer la carrière de l'acteur et Jack Vettriano, qui représente Billy Connolly devant une mer agitée un bras tendu vers le ciel, s'inspire d'un épisode précis de la carrière télévisuelle de l'acteur. En accord avec le city council, ces trois œuvres ont ensuite été reproduites sur les murs de la ville pour être intégrées au Mural Trail. Les fresques Billy Connolly et Dr Connolly, I Presume ont été reproduites d'après les modèles originaux de Byrne et de Vettriano par l'artiste Rogue-One. L'œuvre de Rachel MacLean intitulée Big Yin a quant à elle été réalisée par impression numérique. À travers ces œuvres le jeu d'intermédiation se complexifie puisque les fresques murales ne sont que les reproductions d'œuvres originales elles-mêmes commandées par une tierce organisation. Elles font donc intervenir un dispositif de duplication qui permet de valoriser les œuvres et le sujet représenté en multipliant leurs apparitions. Par la représentation figurative d'abord, par la superposition des œuvres dans la ville ensuite, l'acteur devient de facto un ambassadeur de l'identité locale. En l'intégrant au paysage même de la ville, les pouvoirs publics lui octroient un statut particulier et font de lui l'incarnation d'un ethos glaswegian.

31 Le fait que le projet du City Centre Mural Trail soit conditionné par un objectif de développement touristique signifie qu'il possède une fonction d'incitation pour attirer de nouveaux « consommateurs » culturels. Il devient un outil de séduction capable de matérialiser des symboles et des motifs de façon simple dans l'espace urbain, tout en utilisant des stratégies esthétiques pour favoriser une réaction émotionnelle positive du public. Il est possible dans ce contexte de souligner par exemple la représentation systématique dans les fresques de sujets concrets et reconnaissables, animaux ou humains. Le choix de sujets facilement identifiables permet aux individus de les nommer et de les décrire, ce qui facilite leur intégration au patrimoine commun de la ville et leur diffusion à l'extérieur. La fresque Glasgow's Tiger de l'artiste Klingarton, qui représente de manière détaillée une tête de tigre, s'appuie sur cette stratégie, et son titre marque de manière explicite le lien d'appartenance entre la ville et l'œuvre. Elle en devient un emblème, ce statut étant expressément mis en avant dans le portfolio du City Centre Mural Trail. Le projet tend donc à créer lui-même de nouveaux référents identitaires, historiquement et géographiquement décontextualisés.

Finalement, les œuvres deviennent elles-mêmes des produits culturels répondant à une logique de divertissement. Elles substituent à leurs attributs d'œuvres d'art ceux d'objets, inversion supplémentaire caractéristique du kitsch (voir Genin, 2007). Pour les pouvoirs publics elles s'insèrent dans un système de production et de distribution, tandis que pour les individus elles supposent des stratégies d'acquisition. Le Mural Trail ainsi transformé en produit culturel s'adapte à un modèle économique et social consumériste, distançant un peu plus le projet du street art contestataire qui rejette 
justement ce modèle. Cette "consumérisation" des œuvres et de l'identité locale à laquelle elles renvoient s'exprime à travers leur diffusion médiatique. Les œuvres n'existent plus seulement en elles-mêmes mais à travers les photos qui sont prises d'elles : « À l'ère des médias numériques et des réseaux sociaux toute image de street art est immédiatement mise en ligne, et peut être assimilée, copiée, détournée. " (Genin, $2015, \S 14$ ) Les pouvoirs publics multiplient les supports promotionnels et reproduisent eux-mêmes ces œuvres qui en s'imprimant sur des livrets prennent littéralement la forme d'objets. Diffusées sur Internet, les œuvres se démultiplient au fur et à mesure de l'ajout et du partage de photos entre usagers. Par l'intermédiaire de la photographie et du processus de reproduction de masse qu'elle permet, l'œuvre devient ainsi un bien cessible.

Implicitement, elle s'inscrit alors dans un système de valeur repensé en fonction de la position paradoxale des autorités vis-à-vis du street art. À Glasgow, le street art et les graffitis produits dans un cadre non légal sont considérés de fait comme illégaux et traités comme des actes de vandalisme. Dans le plan stratégique de développement touristique publié en 2007, les autorités locales s'inquiètent ouvertement à ce sujet: "the more immediate problem of litter, graffiti and vandalism threatens to undermine investment in the city» (City Marketing Bureau, 2011). Dans cette optique, les œuvres considérées comme dégradantes sont " légitimement » effaçables et ne " méritent » pas d'être conservées, soit en tant que telles sur les murs, soit par le biais de la photographie. Au contraire les œuvres du Mural Trail deviennent des points de repère qui s'intègrent à la pratique sociale de la visite touristique. Ainsi, elles concrétisent « la conversion du dérangeant en mignon, de l'intolérable en décoratif, de l'effaçable en patrimonialisable » (Genin, 2016). La valeur des œuvres elles-mêmes ne se mesure plus à l'aune de qualités esthétiques intrinsèques, mais en fonction de l'impact médiatique de telle ou telle fresque sur les réseaux sociaux. En tant que produit culturel kitsch, le City Centre Mural Trail renverse donc les hiérarchies normalement établies au sein de la culture urbaine pour recréer son propre système de valeur :

An implicit hierarchy within street art culture tends to give greater significance or credibility to works created without permission, and artists who call themselves street artists but who have never or rarely put up illicit work can sometimes be regarded as less authentic, or as attempting to benefit from street art's fashionability. (Young, 2013)

Finalement, le City Centre Mural Trail peut être lu comme s'inscrivant dans un mouvement global de démocratisation du street art en tant que genre et pratique artistique. Par sa «kitschification» le projet permet donc de satisfaire la demande de nouveaux consommateurs valorisant le street art comme bien culturel.

\section{Conclusion}

L'exploration des enjeux du City Centre Mural Trail permet d'affirmer que l'attrait touristique du projet repose sur sa capacité à diffuser l'image d'une ville moderne et dynamique au sein de laquelle les arts et la culture tiennent une place essentielle. Comme le résume Maria Gravari-Barbas à propos de la ville contemporaine :

On assiste à un glissement progressif de la ville support d'événements festifs ponctuels (ce qui a toujours été le cas dans les villes) vers la ville festive, dans laquelle l'ambiance et l'esthétique de la fête transcendent la conception, la planification, l'aménagement, voire la gestion urbaine. (Gravari-Barbas, 2009) 
36 justement d'attirer l'attention des visiteurs sur cette conception spécifique de la ville et de son identité. Ainsi, il est possible de repenser le kitsch comme opérateur d'attraction culturelle. En délimitant les contours symboliques de la ville et en les rendant accessibles, le kitsch défait l'identité territoriale de sa multiplicité et de sa subjectivité inhérente pour la reconstruire selon une esthétique de la simplicité accessible au plus grand nombre.

Cette étude de cas permet donc de mettre en valeur les enjeux et les tensions d'un projet artistique et politique d'apparence pourtant consensuel. En réalité, le City Centre Mural Trail soulève des problématiques complexes. Alors que la politique menée par les pouvoirs publics pour revaloriser l'environnement urbain permet implicitement à la ville de prendre ses distances avec l'archétype du kitsch écossais, ce rejet d'une identité stéréotypée s'exprime dans des œuvres de street art décoratives et édulcorées qui reposent sur l'assimilation de certaines propriétés du kitsch. Sous un angle d'approche différent, le projet de revalorisation urbaine remet finalement en question la valeur artistique des œuvres tandis que l'affirmation d'une identité locale forte reproduit le schéma de déformation culturelle dont le projet semblait d'abord se distancer. D'un point de vue théorique, c'est bien la question de l'intermédiation institutionnelle qui semble introduire la série de basculements et d'inversions qui font finalement du City Centre Mural Trail un produit culturel. C'est l'effet de cette intervention des pouvoirs publics dans le processus de création artistique qui vient surajouter aux œuvres une dimension transgressive, non dans le sens d'une visée politique et sociale contestataire, mais dans le sens d'une reconfiguration générique et ontologique du street art lui-même et de son rapport à la ville, aux individus et à leur identité. C'est pourquoi cette étude n'a abordé la question du travail de l'artiste et de sa place dans le projet que de manière superficielle. Dans la mesure où on a soulevé l'existence d'un rapport paradoxal des pouvoirs publics au street art à Glasgow, il serait néanmoins envisageable de développer cet élément d'analyse. De même, en considérant que le City Centre Mural Trail répond à un mouvement mondial de revalorisation du street art, il sera intéressant dans les années à venir d'examiner la manière dont l'attitude des pouvoirs publics pourra évoluer vis-à-vis du street art non autorisé.

BIBLIOGRAPHIE

\section{Documents primaires}

\section{Documents imprimés}

Glasgow City Council, City Centre Mural Trail, 2017.

Glasgow City Council, City Centre Mural Trail, Billy Connolly 75th Birthday Special Edition, juin 2017. 
Glasgow City Marketing, Glasgow's Tourism Strategy to 2016, octobre 2007.

Glasgow City Marketing, Glasgow's Tourism and Visitor Plan to 2023, mars 2017.

VisitScotland, The Key Facts on Tourism in 2018, septembre 2019.

VisitScotland, Tourism in Scotland's Regions 2016, août 2017.

\section{Ressources en ligne}

City Centre Mural Trail, <www.citycentremuraltrail.co.uk>.

Glasgow City Council, <https://glasgow.gov.uk/>.

Glasgow City Centre Strategy, <www.glasgowcitycentrestrategy.com>.

People Make Glasgow, <https://peoplemakeglasgow.com>.

VisitScotland, <www.visitscotland.com>.

\section{Documents secondaires}

\section{Ouvrages et articles}

BINKLEY Sam, 2000, « Kitsch as a Repetitive System. A Problem for the Theory of Taste Hierarchy ", Journal of Material Culture, vol. 5, $n^{\circ}$ 2, p. 131-152.

Black Gillian, CRAUfurd SMITH Rachael, KHERIA Smita \& PORTER Gerard, 2015, « Scotland the Brand - Marketing the Myth?», Scottish Affairs, vol. 24, $\mathrm{n}^{\circ}$ 1, p. 47-77.

BLANCHÉ Ulrich, 2015, «Qu'est-ce que le Street art ? Essai et discussion des définitions », Cahiers de Narratologie. Analyse et théorie narratives, $\mathrm{n}^{\circ} 29$. Disponible sur <https://doi.org/10.4000/ narratologie.7397>.

BRown Ian, 2010, From Tartan to Tartanry: Scottish Culture, History and Myth, Édimbourg, Edinburgh University Press.

Goffaux Callebaut Géraldine, GuÉvel Didier \& Seube Jean-Baptiste (dir.), 2017, Droit(s) et Street Art : de la transgression à l'artification, Issy-les-Moulineaux, LGDJ.

GARCíA Beatriz, 2004 « Urban Regeneration, Arts Programming and Major Events ", International Journal of Cultural Policy, vol. 10, $\mathrm{n}^{\circ}$ 1, p. 103-118.

GARCíA Beatriz, 2005, « Deconstructing the City of Culture: The Long-Term Cultural Legacies of Glasgow 1990 », Urban Studies, vol. 42, nºs 5-6, p. 841-868.

GENIN Christophe, 2007, « Le kitsch : une histoire de parvenus », Actes Sémiotiques. Disponible sur <http://epublications.unilim.fr/revues/as/3268> (consulté le 8 janvier 2018).

GENIN Christophe, 2015 « Le street art : de nouveaux principes ? ", Cahiers de Narratologie, n 29. Disponible sur <https://doi.org/10.4000/narratologie.7396>.

GENIN Christophe, 2016, Le Street Art au tournant, Bruxelles, Les impressions nouvelles.

GENIN Christophe, 2017, «Le devenir kitsch : un modèle global pour nos sociétés? », communication lors du colloque Le kitsch : définitions, poétiques, valeurs (Centre culturel international de Cerisy, 22 août 2017) [enregistrement audio en ligne : « Devenir kitsch : un modèle pour nos sociétés », France Culture, 27 septembre 2017, disponible sur <https:// 
www.franceculture.fr/conferences/maison-de-la-recherche-en-sciences-humaines/quand-lekitsch-devient-un-modele-de-societe>].

GRAVARI-BARBAS Maria, 2009, « La "ville festive" ou construire la ville contemporaine par l'événement », Bulletin de l'Association de géographes français, vol. 86, n 3, p. 279-290. Disponible sur $<$ https://doi.org/10.3406/bagf.2009.2673>.

HALl Tim \& ROBERTSON Iain, 2001, « Public Art and Urban Regeneration: Advocacy, Claims and Critical Debates », Landscape Research, vol. 26, n 1, p. 5-26.

JEANNIER Fabien, 2008 «Culture et régénération urbaine : le cas de Glasgow », Géoconfluences. Disponible sur <http://geoconfluences.ens-lyon.fr/doc/typespace/urb1/MetropScient7.htm> (consulté le 8 janvier 2018).

Keating Michael, 1988, The City That Refused to Die: Glasgow: The Politics of Urban Regeneration, Aberdeen, Aberdeen University Press.

LEYDIER Gilles, 2005, « Glasgow réinventée ?», dans R. Findlay, W. Findlay et T. Tran (éds), Vivre la Ville en Écosse: Towns and Town Life in Scotland, Tours, Presses universitaires François Rabelais, p. 241-254.

MACLELLAN Rory \& SMITH Ronnie (éds), 1998, Tourism in Scotland, Londres, International Thomson Business.

McCoRmick Carlo, 2010, Trespass : une histoire de l'art urbain illicite, édité par E. Seno, Paris, Taschen. PADDISON Ronan, 1993, « City Marketing, Image Reconstruction and Urban Regeneration », Urban Studies, vol. 30, $\mathrm{n}^{\circ}$ 2, p. 339-350.

PADDISON Ronan, 2013, «Ethnic Diversity, Public Space and Urban Regeneration », dans M. E. Leary et J. McCarthy, The Routledge Companion to Urban Regeneration, Londres, Routledge, p. 263-272.

RIFFAUD Thomas \& RECOURS Robin, 2016, « Le street art comme micro-politique de l'espace public : entre "artivisme" et coopératisme ", Cahiers de Narratologie, $\mathrm{n}^{\circ} 30$. Disponible sur $<\mathrm{https}$ :// doi.org/10.4000/narratologie.7484>.

SHARP Joanne, Pollock Venda \& PADDISON Ronan, 2005, « Just Art for a Just City: Public Art and Social Inclusion in Urban Regeneration », Urban Studies, vol. 42, $\mathrm{n}^{\text {os }}$ 5-6, p. 1001-1023.

STAHL Johannes, 2009, Street Art, Cologne, H. F. Ullmann Publishing.

VisConTI Luca M., SHERRY John F., BORGHINI Stefania \& ANDERSON Laurel, 2010, « Street Art, Sweet Art? Reclaiming the "Public" in Public Place ", Journal of Consumer Research, vol. 37, n 3 , p. 511-529.

Young Alison, 2013, Street Art, Public City: Law, Crime and the Urban Imagination, Londres, Routledge.

\section{Articles de presse}

AsH Lucy, « Why Is Glasgow the UK's Sickest City? », BBC News, 5 juin 2014. Disponible sur <www.bbc.com/news/magazine-27309446> (consulté le 8 janvier 2018).

"Following in the Footsteps of Banksy, Glasgow “Taggers" Call for Legal Spaces to Display Artworks ", The Herald, 30 juillet 2017. Disponible sur <www.heraldscotland.com/news/ 15441089.Following_in_the_footsteps_of_Banksy__Glasgow___39_taggers__39__call_for_legal_spaces_to_display_artworks/ $>$ (consulté le 8 janvier 2018). 
SMITH Ken, « At Large: It's More than the Writing That's on the Walls for Glasgow », The Herald, 30 juin 2015. Disponible sur <www.heraldscotland.com/opinion/ 13414991.At_Large__It_s_more_than_the_writing_that_s_on_the_walls_for_Glasgow/> (consulté le 8 janvier 2018).

« Tartan Kitsch Has Its Place ... but It's Not Modern Scotland », The Herald, 17 octobre 2009. Disponible sur <www.heraldscotland.com/default_content/12609835.tartan-kitsch-has-its-placebut-its-not-modern-scotland/> (consulté le 8 janvier 2018).

\section{NOTES}

1. Le titre fait référence à une comédie américaine à succès sortie en 1989 dans laquelle un inventeur réduit accidentellement la taille de ses enfants et de ceux de ses voisins à quelques millimètres. Dans le film on peut par la suite le voir muni d'une loupe à la recherche des enfants.

2. Le Mural Trail est inclus dans la liste des activités conseillées sur le site du guide officiel de la ville, People Make Glasgow à l'adresse <https://peoplemakeglasgow.com/things-to-do/city-centremural-trail>.

3. L'initiative a été primée aux Scottish Arts and Business Awards en 2017.

4. «Following in the Footsteps of Banksy, Glasgow "Taggers" Call for Legal Spaces to Display Artworks », The Herald, 30 juillet 2017.

5. Du titre d'un article publié sur le site BBC News : "Why Is Glasgow the UK's Sickest City?», 5 juin 2014.

\section{RÉSUMÉS}

Cet article propose une étude de cas du Glasgow City Centre Mural Trail, une initiative de la municipalité de Glasgow lancée en 2014 qui vise à financer des fresques murales à des fins de réhabilitation urbaine et de promotion touristique. À travers l'analyse des œuvres et des documents produits autour du projet, il s'agit d'examiner le choix du street art comme modalité d'intervention dans l'espace urbain et comme vecteur d'une identité locale résolument urbaine portée par une imagerie distincte des représentations populaires de l'Écosse. Dans un second temps, en s'appuyant notamment sur les travaux de Christophe Genin sur les rapports entre kitsch et art urbain, cette étude explore les implications de l'intermédiation des pouvoirs publiques sur l'activité artistique. Le processus de patrimonialisation par lequel le Mural Trail s'intègre dans l'espace urbain soulève ainsi la question des attributs du street art lui-même et de l'identité glaswegian devenue objet de représentation et de reproduction. Il s'agit finalement de démontrer qu'en tant que décor urbain et produit d'attraction touristique le projet s'approprie en réalité certains critères $\mathrm{du}$ « kitsch » qu'il parait au premier abord contester.

This paper examines the Glasgow City Centre Mural Trail, a project launched by the Glasgow city council in 2014. The city council financially supports the production of murals by local street artists in order to rejuvenate the city and promote local tourism. Relying on an analysis of the artworks themselves and related official documents, this article looks at the choice of street art as a means to reshape Glasgow's urban landscape and promote a distinctive image of the city 
significantly different from traditional representations of Scotland. Building on the work of Christophe Genin on kitsch and street art, this article then explores the artistic and symbolic issues raised by the project. As public authorities provide legitimacy to the artworks, which become fully part of Glasgow's urban environment, their representation of Glaswegian identity as well as the nature of street art as an artistic practice are put into question. By becoming a tourist attraction, the Mural Trail thus seems to assume a "kitsch" dimension even as it apparently challenges "kitsch".

\section{INDEX}

Mots-clés : industrie du tourisme, identité territoriale, politiques culturelles (Glasgow), art urbain, street art, Christophe Genin

Keywords : tourism industry, territorial identity, cultural politics (Glasgow), urban art, street art, Christophe Genin

\section{AUTEUR}

\section{EMERENCE HILD}

SEARCH, Université de Strasbourg.

Emrence Hild est doctorante contractuelle en civilisation écossaise à l'Université de Strasbourg (EA 2325) et prépare une thèse sur Le Scottish National Party et l'identité nationale écossaise, 1934-2015. 